\title{
TWO NEW RECORDS OF KATYDIDS (ORTHOPTERA: TETTIGONIIDAE: CONOCEPHALINAE) FROM THE DOMINICAN REPUBLIC, HISPANIOLA
}

\author{
Daniel E. Perez-Gelabert \\ Integrated Taxonomic Information System (ITIS) and Department of Entomology, United States \\ National Museum of Natural History, Smithsonian Institution, P.O. Box 37012, Washington, \\ DC 20013-7012, USA.perezd@si.edu
}

\begin{abstract}
The conocephaline katydids Erechthis gundlachi Bolívar, 1888 and Pyrgocorypha uncinata (Harris, 1841) are recorded for the first time from the Dominican Republic and the island of Hispaniola. Both species were previously known from Cuba, although $P$. uncinata is also distributed in the southeastern United States.
\end{abstract}

Keywords: Katydids, Orthoptera, faunistics, Dominican Republic, Hispaniola, Greater Antilles.

Título: Dos nuevos registros de esperanzas (Orthoptera: Tettigoniidae: Conocephalinae) para la República Dominicana, La Hispaniola.

\section{RESUMEN}

Se reportan por primera vez para la República Dominicana y La Hispaniola las esperanzas conocefalinas Erechthis gundlachi Bolívar, 1888 y Pyrgocorypha uncinata (Harris, 1841). Ambas especies eran previamente conocidas de Cuba, aunque $P$. uncinata también se distribuye en el sureste de los Estados Unidos.

Palabras clave: Esperanzas, Orthoptera, fauna, República Dominicana, Hispaniola, Antillas Mayores.

\section{INTRODUCTION}

The Neotropical fauna of katydids is very diverse containing over 1800 known species, with possibly as many more species yet to be discovered in the region (Naskrecki, 2000). Despite their large size, ubiquitous presence and important ecological roles, the taxonomy of the family Tettigoniidae is still preliminary. In Hispaniola the entomofauna in general is poorly known as it has been haphazardly studied mostly by visiting entomologists. Hispaniolan katydids in particular have never been collected and studied in a systematic manner including wide-ranging collections from throughout the varied geography of this island. Only 23 species in 11 genera of Tettigoniidae have so far been recorded, 14 (60.9\%) of these species being endemic to Hispaniola (Perez-Gelabert, 2008). Judging from material already in collection, the total number of katydid species in the island should be at least double that number. Hispaniola shares a portion of its katydid species with the other Greater Antilles where they appear to be only slightly better documented.

\section{OBJECTIVE}

- To provide first time records from the Dominican Republic and the island of Hispaniola for the katydids Erechthis gundlachi Bolívar, 1888 and Pyrgocorypha uncinata (Harris, 1841). 


\section{MATERIAL AND METHODS}

Most of the material examined was collected as part of the Hispaniolan orthopteroids project during night searches of the vegetation using lights throughout many localities of the Dominican territory. Cuban specimens of both species were directly examined in the entomological collection of the Instituto de Ecología y Sistemática, La Habana, Cuba. Most specimens of E. gundlachi studied here are deposited at the USNM, excepting series of 4 males and 4 females that will be deposited in the ANSP, IIBZ and MNHNSD collections. The specimens of $P$. uncinata were borrowed from Dr. Robert E. Woodruff (FSCA). Excepting the live picture, habitus photographs were taken using the Visionary Digital (TM) BK Lab imaging system outfitted with a Canon Mark II 5D.

Institutional abbreviations. ANSP - Academy of Natural Sciences, Philadelphia, Pennsylvania, USA. FSCA - Florida State Collection of Arthropods, Gainesville, Florida, USA. IES - Instituto de Ecología y Sistemática, La Habana, Cuba. IIBZ - Instituto de Investigaciones Botánicas y Zoológicas, Universidad Autónoma de Santo Domingo, Dominican Republic. MNHNSD Museo Nacional de Historia Natural de Santo Domingo, Dominican Republic. USNM - United States National Museum of Natural History, Smithsonian Institution, Washington, DC, USA.

\section{Subfamily Conocephalinae Burmeister, 1838 \\ Tribe Agraeciini Redtenbacher, 1891 \\ Erechthis gundlachi Bolívar, 1888}

\section{Figure 1, A-C}

Recognition. Straw-yellowish to light brown sometimes greenish conocephaline katydids. Medium size (from tip of head to tip of wings: 38-43 mm males, $43-54 \mathrm{~mm}$ females), body slender, with a spiny fastigium that projects slightly beyond the head vertex and curves downward at the tip. Posterior lobes of pronotum somewhat rounded, posterior pronotal margin flat. A distinctive dark brown band runs on the dorsal field from tip of fastigium and over pronotum to tegminae. Wings fully-developed with rounded tips. Female ovipositor a curving sword ending in a fine point short of the wing tips.

Comments. The genus Erechthis Bolívar, 1888 is monotypic and originally described from specimens collected in eastern Cuba. Only a few years after its description, Redtenbacher (1891) gave its distribution as Cuba and Puerto Rico and this was repeated by authors like Karny (1912). I have not been able to find any citation of Puerto Rican specimens in the literature. There is one specimen of a genus similar to Erechthis in the collection of the University of Puerto Rico, Mayagüez. Local photographers Alejandro Sánchez and Alfredo Colón who have pages of insect photos on the Internet have never photographed this katydid. If this species is indeed found in Puerto Rico, it seems to be rare.

Erechthis was included in the checklist of Hispaniolan arthropods by Perez-Gelabert (2008) without certainty on the species identity. As shown by the large number of specimens examined here which were collected in 14 provinces of the Dominican Republic, this species is common in shrubs and small trees from near the coast to over one thousand meter elevation. It has a preference for moderately wet areas and vegetation at the edge of trails and small roads. Adults are found throughout the year. Erechthis gundlachi was frequently observed eating flowers/ pollen of various plants early at night. Also demonstrating their predaceous preferences, in locality RD-202 (La Enea, near Higuey, La Altagracia prov.) a nymph of E. gundlachi was found eating a Phyllophaga beetle. In another case one individual was encountered eating a stick insect. 

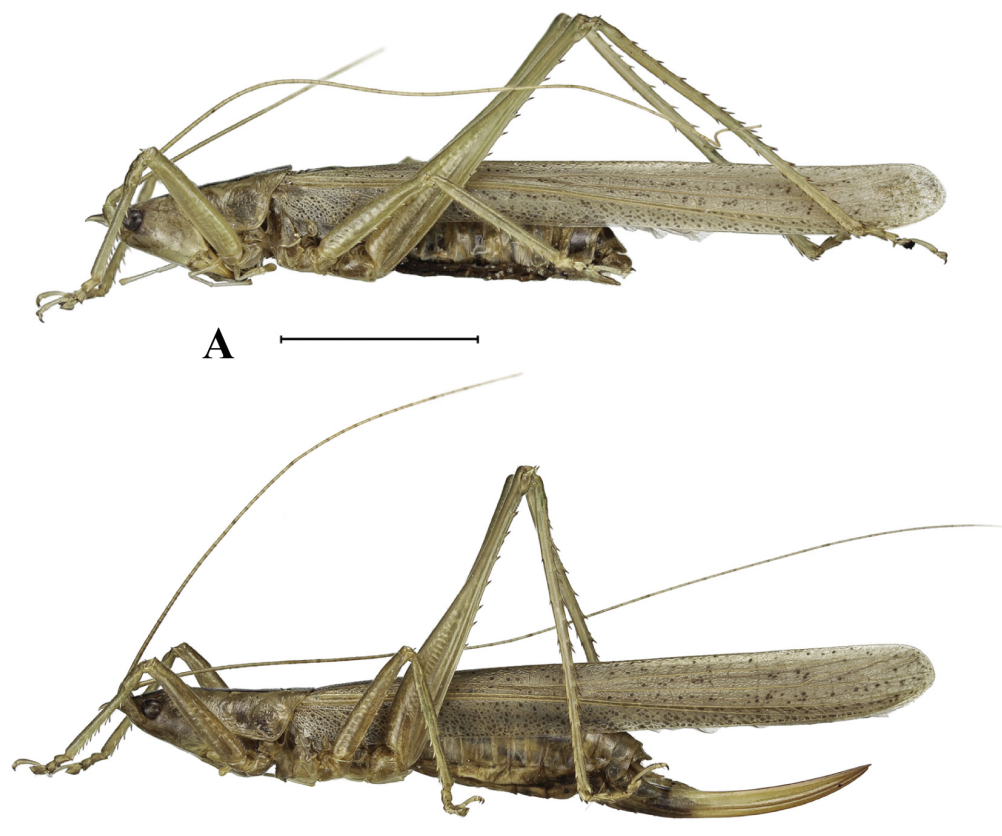

B

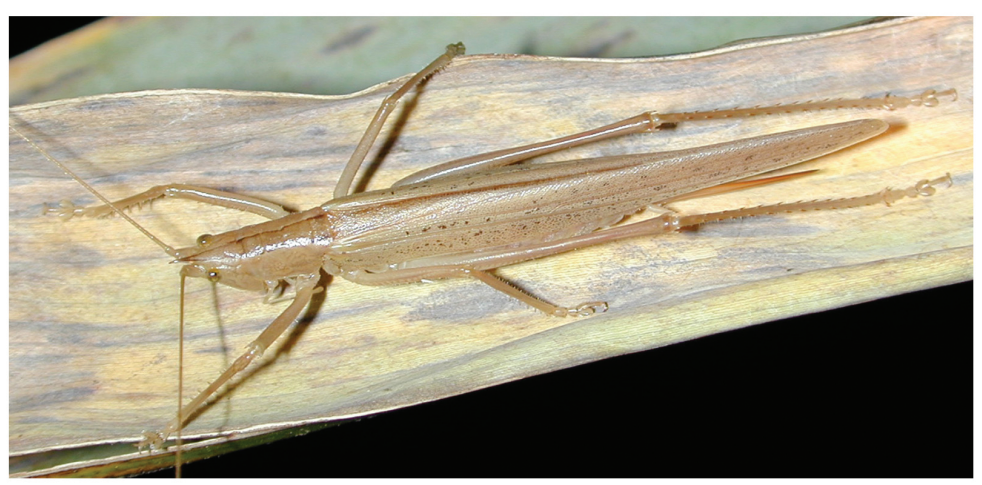

C 
General distribution. Cuba and Hispaniola.

Material examined. 1 male, RD-002 DOMINICAN REPUBLIC, 13.i.02 Mano Matuey, San Cristóbal Prov., $\sim 100 \mathrm{~m}$ up from road, (coffee plantation mixed w/ other crops), $460 \mathrm{~m}, 367-834$ mE 2045-450 mN. D. Otte, D. Perez, R. Bastardo; 1 female, RD-014 DOMINICAN REPUBLIC, 18.i.02 Down from Monteada Nueva, Barahona Prov., by the road, 970 m, 261-199 mE 2005848 mN. D. Otte, D. Perez, R. Bastardo, S. Medrano, B. Hierro; 2 males, RD-020 DOMINICAN REPUBLIC, 23.i.02 Jardín Botánico Nacional, Reserva Forestal, Santo Domingo, Distrito Nacional, 18²9.60’N 6957.19'W. D. Otte, D. Perez, R. Bastardo, S. Medrano; 1 male, RD021 DOMINICAN REPUBLIC, 26-27.i.02 Arroyazo, Reserva Científica Ebano Verde, La Vega Prov., 990 m, 1902.27’N 70 32.64'W. D. Perez, R. Bastardo, B. Hierro; 2 males 1 female, RD-042 DOMINICAN REPUBLIC, Arroyazo, Reserva Científica Ebano Verde, La Vega Prov., $19^{\circ} 01.945^{\prime} N$ 70³2.593'W, 9-10.vii.2002, 3,500 ft. D. Perez, B. Hierro, R. Bastardo; 17 males 18 females, RD-044 DOMINICAN REPUBLIC, La Sal, Reserva Cientifica Ebano Verde, La Vega Prov., 11-12.vii.2002, 1904.101'N 70³4.089'W, 1,043 m, D. Perez, B. Hierro, R. Bastardo; 1 males 2 females, RD-045 DOMINICAN REPUBLIC, Paso de la Perra, nr. La Ciénaga, La Vega Prov., 1904.576'N 7049.623'W, 16.vii.2002. D. Perez, B. Hierro, R. Bastardo, S. Medrano, H. Takizawa; 1 males 3 females, RD-049 Los Tablones, Parque Nacional Armando Bermúdez, La Vega Prov., 1903.308’N 7053.049’W, 19.vii.2002, D. Perez, B. Hierro, R. Bastardo, S. Medrano, H. Takizawa; 1 male 2 females, RD-051 DOMINICAN REPUBLIC, Alto del Rancho, Loma Guaconejo, M. T. Sánchez Prov., 170 m, 24-25.vii.2002, 19¹8.752'N 6956.663'W, D. Perez, B. Hierro, R. Bastardo; 1 female, RD-055 DOMINICAN REPUBLIC, $2 \mathrm{~km} \mathrm{~N}$ Bayahibe, La Altagracia Prov., 31.vii.2002, $18^{\circ} 23.423^{\prime} N$ 6850.453'W, D. Perez, R. Bastardo, B. Hierro; 1 male, RD-056 DOMINICAN REPUBLIC, Pueblo Nuevo, Baní, Peravia Prov., dry scrub, $18^{\circ} 17.672^{\prime} N$ 70¹9.922’W, 16.xi.2002, D. Perez, R. Bastardo, B. Hierro. (night); 1 female, RD-62 DOMINICAN REPUBLIC, $\sim 5 \mathrm{~km}$ W of Las Americas Airport, nr. LADOM, Santo Domingo Prov., $18^{\circ} 28.158^{\prime}$ N 6943.601'W, 19.xi.2002, D. Perez, R. Bastardo, B. Hierro. (night); 1 male, RD-064 DOMINICAN REPUBLIC, Parque Mirador del Norte, Santo Domingo Prov., 96 m, $18^{\circ} 31.387^{\prime} N$ 6955.497’W, 21.xi.2002, D. Perez, R. Bastardo, B. Hierro. (night); 2 males, RD-072 DOMINICAN REPUBLIC, $\sim 5 \mathrm{~km} \mathrm{~N}$ of La Colonia, San Cristóbal Prov., 566 m, $18^{\circ} 31.167^{\prime} \mathrm{N} 70^{\circ} 16.740^{\prime} \mathrm{W}, 26 . x i .2002$, D. Perez, B. Hierro, R. Bastardo. (night); 2 males 1 female, RD-073 DOMINICAN REPUBLIC, La Colonia, San Cristóbal Prov., 773 m, 18³1.167’N 70¹6.740’W, 26.xi.2002, D. Perez, B. Hierro, R. Bastardo. (night); 1 male 1 female, RD-083 DOMINICAN REPUBLIC, Juan Dolio, San Pedro de Macorís Prov., sea level, nr. $18^{\circ} 26.35^{\prime} N 6^{\circ} 24.06^{\prime} \mathrm{W}, 6 . x i i .2002$, D. Perez, R. Bastardo. (night); 5 males, 3 females, RD087 DOMINICAN REPUBLIC, Way down from Palos Grandes, NE of S. J. de Ocoa, Ocoa Prov., 1,440 m, $18^{\circ} 37.283^{\prime} \mathrm{N} 70^{\circ} 31.481^{\prime} \mathrm{W}$, 8.xii.2002, D. Perez, R. Bastardo. (night); 1 male, RD-091 DOMINICAN REPUBLIC, Sierra Prieta, Santo Domingo Prov., 133 m, 18³9.010’N 6958.409’W, 18.iii.2003, D. Perez, R. Bastardo, B. Hierro. (night); 5 males 7 females, RD092 DOMINICAN REPUBLIC, Blanco (near hydroelectric), Bonao, Monseñor Nouel Prov., $18^{\circ} 52.946^{\prime} N$ 70³0.337’W, 19.iii.2003, D. Perez, R. Bastardo, B. Hierro. (night); 5 males, RD098 DOMINICAN REPUBLIC, 4 km W of Las Americas Airport, Santo Domingo Prov., nr. sea level, $18^{\circ} 28.110^{\prime} \mathrm{N}$ 6943.358’W, 22.iii.2003, D. Perez, B. Hierro, S. Medrano (night); 1 male 2 females, RD-122 DOMINICAN REPUBLIC, La Jarda (monte abajo), Padre Las Casas, Azua Prov., 1844.094’N 7052.208’W, 5.iv.2003, D. Perez, B. Hierro, S. Medrano, D. Veloz (night); 1 female, RD-127 DOMINICAN REPUBLIC, Diferencia, $1 \mathrm{~km}$ from caseta, PNAB, Santiago Prov., 750 m, 19¹6.080’N 71ํ⒉763’W, 8.iv.2003, D. Perez, R. Bastardo, B. Hierro. (night); 1 female, RD-128 DOMINICAN REPUBLIC, around caseta La Sierrecita, PNAB, Santiago Prov., 752 m, 19¹4.889’N 71ํ⒋735’W, 9.iv.2003, D. Perez, R. Bastardo, B. Hierro. (day-night); 7 males 8 females, RD-140 DOMINICAN REPUBLIC, $\sim 1 \mathrm{~km}$ SE caseta no. 1, Parque Nacional Sierra de Bahoruco, Independencia Prov., 18¹5.771’N 71³2.233’W, 1,153 
m, 4.vii.2003, D. Perez, R. Bastardo, B. Hierro. (day/night); 1 male, RD-148 DOMINICAN REPUBLIC, $\sim 100 \mathrm{~m}$ up from El Sitio del Agua, cloud forest, N Los Bolos, Sierra de Neiba, Independencia Prov., 18³9.339’'N 7139.279’W, 1,520 m, 9.vii.2003, D. Perez, R. Bastardo, B. Hierro. (day/night); 2 males 9 females, RD-151 DOMINICAN REPUBLIC, La Sal, Reserva Ebano Verde, La Vega Prov., $1^{\circ} 04.101^{\prime} \mathrm{N}$ 70 34.089’W, 1,043 m, 12.vii.2003, D. Perez, R. Bastardo, B. Hierro. (night); 2 males 3 females, RD-154 DOMINICAN REPUBLIC, Busú, El Curro, Sierra Martín García, Azua Prov., 18¹7.819’N 7057.287’W, 771 m, 16-17.vii.2003, D. Perez, R. Bastardo, B. Hierro. (day/night); 1 female, RD-155 DOMINICAN REPUBLIC, Charquito Prieto, El Curro, Sierra Martín García, Azua Prov., 18¹8.324'N 7057.176’W, 731 m, 17-18.vii.2003, D. Perez, R. Bastardo, B. Hierro. (day/night); 3 males 3 females, RD157 DOMINICAN REPUBLIC, Los Tablones, Parque Armando Bermúdez, La Vega Prov., $1^{\circ} 03.308^{\prime} \mathrm{N} 70^{\circ} 53.049^{\prime} \mathrm{W}, 1,270 \mathrm{~m}, 23 . v i i .2003$, D. Perez, R. Bastardo, B. Hierro. (night); 2 females, RD-179 DOMINICAN REPUBLIC, La Laguna, N. El Valle, Samaná Prov., 28-29. xi.2003, $54 \mathrm{~m}, 1^{\circ} 15.007^{\prime} \mathrm{N} 6^{\circ} 18.471^{\prime} \mathrm{W}, \mathrm{D}$. Perez, R. Bastardo, A. Francisco. (day/night); 4 females, RD-181 DOMINICAN REPUBLIC, Loma Quita Espuela, halfway to peak, 616 m, 19²0.912'N 7008.941'W, 3.xii.2003, D. Perez, R. Bastardo, A. Marmolejos. (day/night); 1 female, RD-182 Loma Quita Espuela, firme de loma, S. F. de Macorís, [Duarte] Prov., $19^{\circ} 21.101^{\prime} \mathrm{N} 70^{\circ} 08.930^{\prime} \mathrm{W}, 715$ m, 3-4.xii.2003, D. Perez, R. Bastardo, A. Marmolejos (day/ night); 1 male 2 females, RD-184 DOMINICAN REPUBLIC, Trail to peak and Centro SOECI, Pico Diego de Ocampo, Santiago Prov., 918 m, 5.xii.2003, D. Perez, R. Bastardo, A. Marmolejos. (day/night); 1 female, RD-199 DOMINICAN REPUBLIC, Boca de Yuma, P. N. Del Este, La Altagracia Prov., 20 m, 18²1.875'N 68³7.081'W, 16-17.xii.2003, D. Perez, R. Bastardo (day/ night); 2 males 3 females, RD-202 DOMINICAN REPUBLIC, La Enea, $15 \mathrm{Km}$ W of Higüey, La Altagracia Prov., 18 $39.415^{\prime} \mathrm{N}$ 68 51.129'W, 100 m, 18.xii.2003, D. Perez, B. Hierro, R. Bastardo (night); 1 male 1 female, RD-203 DOMINICAN REPUBLIC, Rd. El Seibo - Miches, El Seibo Prov., $18^{\circ} 55.435^{\prime}$ N 6907.065'W, 18.xii.2003, D. Perez, B. Hierro, R. Bastardo (night); 1 male 1 female, RD-219 DOMINICAN REPUBLIC, Sierra Prieta, Villa Mella, Santo Domingo Prov., 142 m, 18 $8^{\circ} 38.925^{\prime} \mathrm{N}$ 6958.303'W, 12.iv.2004, D. Perez, B. Hierro, R. Bastardo. (n); 3 females, DOMINICAN REPUBLIC, alrededores Casa Federación de Campesinos, Blanco, Monseñor Nouel Prov., 680 m, 1-2.iii.2003, 341-380 mE 2088-015 mN, R. Bastardo; 1 female, DOMINICAN REPUBLIC, San Cristóbal Prov., 3 km N La Colonia, Mano Matuey, 7.ix.2008, D. Perez, B. Hierro, R. Bastardo, S. Medrano; 1 male 1 female, DOMINICAN REPUBLIC, Guaraguao, Parque Nacional del Este, $18^{\circ} 19.893^{\prime} \mathrm{N}$ 68 $48.715^{\prime} \mathrm{W}, 20 . v i i i .2011$, D. Perez, S. Medrano, A. Hilario (day + UV lights). One borrowed Cuban specimen with the following data was also directly compared to the Hispaniolan specimens: "Rio Seco, San Carlos Est. Guantanamo Cuba 1 July 1918 CollC. T. Ramsden" §̋ Erechthis gundlachi Det. P. Alayo 1964. Face down label: CZ-ACC 7.600290 [IES].

Tribe Copiphorini Karny, 1912

Pyrgocorypha uncinata (Harris, 1841)

Figure 2, A-B

Recognition. Greenish brown to light brown colored rather robust conocephaline katydids. Medium size (from tip of head to tip of wings: $53 \mathrm{~mm}$ the male, $54-58 \mathrm{~mm}$ the females). Both sexes fully-winged with tegminae tips rounded. Fastigium projecting prominently beyond the eyes, triangular viewed from above, flat on top, with a spiny downward curving tip, and a large basal tooth on its lower side. Female ovipositor a large mostly straight sword ending in a fine tip, not reaching tips of tegmina. The common name in English given to this katydid is "hookfaced conehead". 
Comments. The genus Pyrgocorypha Stål, 1873 includes 6 species distributed in tropical America and 10 species in the Oriental region (Eades et al., 2011). At least in Hispaniolan territory $P$. uncinata seems to be rare and so far has only been found in the montane locality of Río Limpio, Elías Piña Prov. Specimens were collected when attracted to a UV lighted sheet.

General distribution. Southeastern United States, Cuba and Hispaniola.

Material examined. 1 male, 6 females, DOMINICAN REPUBLIC, Prov. Elias Piña, Rio Limpio, 26-27-iv-2000, 2400 ft., blacklight trap, R. E. Woodruff, T. J. Henry. [FSCA]. These specimens were directly compared to one borrowed Cuban specimen with the following data: "Jovellanos, E. Exp. Caña iv-1965. Col. I Garcia Pyrgocorypha uncinata § Det. I. Garcia 1965”. Face down label: CZ-ACC 7.600321 [IES].

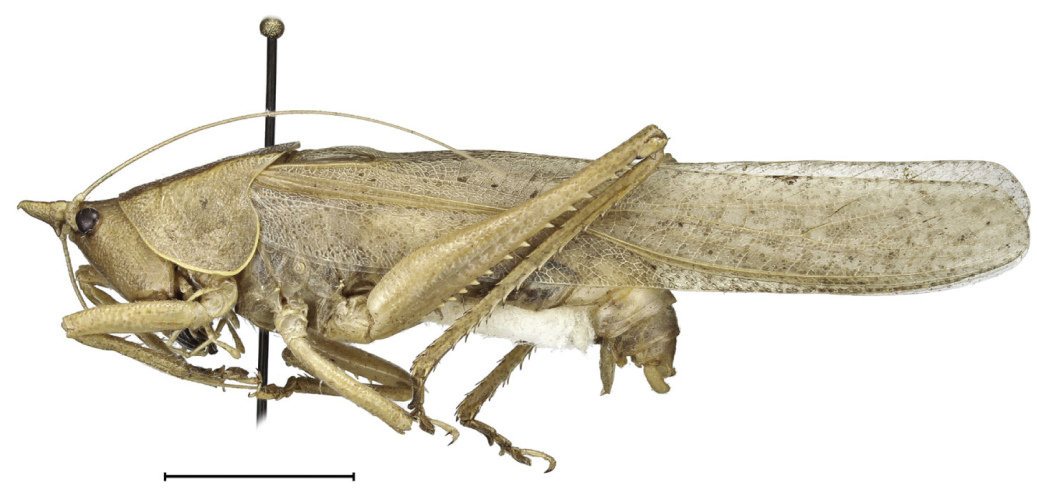

A

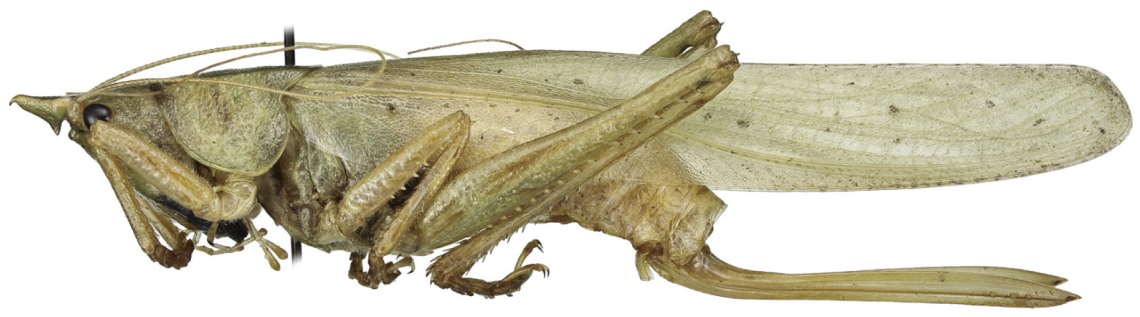

B 


\section{ACKNOWLEDGMENTS}

Dr. Robert E. Woodruff (FSCA) loaned me the Dominican Pyrgocorypha uncinata specimens. Dr. Alejandro Segarra (University of Puerto Rico, Mayagüez) checked for specimens of Erechthis in their collection. Karolyn Darrow (Department of Entomology, Smithsonian Institution) produced the excellent habitus pictures. Ruth Bastardo (IIBZ), Brígido Hierro (Departamento de Vida Silvestre), Denia Veloz and Sardis Medrano (then at MNHNSD) were helpful participants of the orthopteroids project in the Dominican Republic. In Cuba, Rayner Nuñez, Elba Reyes (IES), Esteban Gutiérrez (Museo Nacional de Historia Natural, La Habana, Cuba) and Sheyla Yong (Universidad de La Habana), made possible my successful visit, the study of Cuban collections and taking specimens on loan.

\section{LITERATURE CITED}

Eades, D. C., D. Otte, M. M. Cigliano and H. Braun. 2011. Orthoptera Species File Online (OSF). Versión 5.0/5.0. Disponible en http://orthoptera.speciesfile.org/HomePage/Orthoptera/ HomePage.aspx (Consulted April, 2014).

Karny, H. 1912. Genera Insectorum. Orthoptera, Fam. Locustidae, subfam. Agraeciinae. Part 141, P. Wytsman, Brussels, 47 pp.

Naskrecki, P. 2000. Katydids of Costa Rica: vol. 1. Systematics and bioacoustics of the conehead katydids (Orthoptera: Tettigoniidae: Conocephalinae sensu lato). The Orthopterists' Society and the Academy of Natural Sciences of Philadelphia, 164 pp.

Perez-Gelabert, D. E. 2008. Arthropods of Hispaniola (Dominican Republic and Haiti): a checklist and bibliography. Zootaxa, 1831: 1-530.

Redtenbacher, J. 1891. Monographie der Conocephaliden. Verhandlungen der kaiserlichköniglichen zoologisch-botanischen Gesellschaft in Wien, 41: 315-562.

[Recibido: 29 de abril, 2014. Aceptado para publicación: 28 de agosto, 2014] 